\title{
Estudio comparativo del nivel de actividad física, estado nutricio y obesidad abdominal en profesores de educación física dela UniversidadAutónomade Sinaloa y la Universidad de Huelva
}

\section{Comparative study of the physical activity level, nutrition state and abdominal obesity in physical education professors from the University Autonomus of Sinaloa and Huelva University}

\author{
*Javier Arturo Hall López, **Paulina Yesica Ochoa Martínez, ***Pedro Sáenz-López Buñuel, \\ ****Luis Roberto Monreal Ortiz \\ *Universidad Autónoma de Baja California (México), **Universidad Autónoma de Chihuahua (México), ***Universidad de Huelva (España), \\ ****UniversidadAutónoma de Sinaloa(México)
}

Resumen: El propósito de este trabajo fue comparar la prevalencia del nivel de actividad física, estado nutricio y obesidad abdominal en profesores universitarios de educación física de la Universidad Autónoma de Sinaloa (UAS) México y la Universidad de Huelva (UHU) España. Fueron estudiados 52 docentes de amabas universidades, clasificando el nivel de actividad física mediante el cuestionario internacional de actividad física IPAQ versión corta, se tomaron medidas antropométricas (peso, talla y circunferencia umbilical) para determinar el índice de masa corporal y la obesidad abdominal. Resultando con combinación similar de nivel de actividad física alta y moderada, la prevalencia combinada de sobrepeso y obesidad fue considerablemente mayor en los profesores de la UAS (90.6\%) que en profesores de la UHU (37.5\%), de igual forma la obesidad abdominal (30.6\% y 12.5\% respectivamente), mostrando sujetos físicamente activos, pero por otro lado, en los profesores de genero masculino se observó alta prevalencia combinada de sobrepeso y obesidad incluso mayor al promedio de adultos mexicanos y españoles. Concluyendo que en estos sujetos es necesario tomar medidas, como poner en marcha programas de nutrición orientados a frenar el problema del sobrepeso y obesidad. Palabra clave: actividad física, estado nutricio, obesidad Abdominal, profesores de educación física.

Abstract: The aim of this work was to compare the prevalence of physical activity level, nutrition state and abdominal obesity in physical education professors from the University Autonomus of Sinaloa (UAS) Mexico and Huelva University (UHU) Spain, 52 professors were studied, in order to classify the physical activity level the International Physical Activity Questionannaire (IPAQ) short version was applied. To determine the nutrition state and abdominal obesity, anthropometric measurements were taken (weight, stature and waist circumference). Resulting whith similar prevalence of high and moderate physical activity, the prevalence of combined overweight and obesity was considerly higher in UAS profesors (90.6\%) than UHU professors (37.5\%), the same way in the prevalence of abdominal obesity $30.6 \%$ to $12.5 \%$. The findings of this study shows subjects physically active, but for other side this study shows in both university professors high prevalence of overweight and obesity even higher than their respective people average country. We can conclude that in these subjects efforts should be made to develop nutritions programs to help stem rates of overweight and obesity.

Key words: physical activity, nutrition state, abdominal obesity, physical education professors.

\section{Introducción}

La Organización Mundial de la Salud(OMS) en 1997 revela que el sobrepeso y la obesidad afectan a más de la mitad de la población de muchas naciones, sugiriendo que se debe atender la problemática para evitar el incremento de enfermedades crónico no trasmisibles asociadas con estos. Según datos de la Organización Panamericana de la Salud, las enfermedades crónicas representan el 60\% de las causas de muerte a nivel global, cifra que ascenderá a 73\% para el año 2020. Las sociedades modernas, urbanas en su mayoría, parecen converger hacia el consumo de alimentación alta densidad de energía, ricas en grasas e hidratos de carbono y bajas en fibra, que se asocian con una vida cada vez más sedentaria. Estos cambios magnifican los problemas resultantes de la transición epidemiológica y hacen que la edad en que aparecen las enfermedades crónicas asociadas con la alimentación, sea cada vez más temprana y en grupos de menores ingresos (Lynch et al. 1996; Lang \& Ducimetiere, 1995).

Los hábitos de vida sedentarios constituyen uno de los grandes factores de riesgo que explican las proporciones epidémicas actuales de las enfermedades no transmisibles. Estos hábitos sedentarios son influidos tal vez por un perfil de violencia cada vez mayor en las zonas urbanas, que inhibe a sus habitantes de actividades tales como caminar, correro andarenbicicleta, y que se suma ala carencia de lugares apropiados

\footnotetext{
Fecha recepción: 29-01-08 - Fecha envío revisores: 04-04-08 - Fecha de aceptación: 18-09-08 Correspondencia: Javier Arturo Hall López

Rio Mocorito y Monclova S/N, Col. Ex ejido Coahuila

C.P. 21280 Mexicali, Baja California, México

E-mail: javihall@yahoo.com.mx
}

para las prácticas de algún deporte. La inactividad física representa mucho más que la ausencia de actividad, ya que generalmente se asocia a costumbres que involucran incrementar el consumo de alimentos con alto contenido energético. La correlación entre horas frente a la TV y aumento en el consumo de alimentos de alta densidad energética, ha sido demostrada en todos los grupos etareos. El sedentarismo se constituye en uno de los grandes factores de riesgo que explica las proporciones epidémicas actuales de las enfermedades no transmisibles. $76 \%$ de todas las defunciones en el continente americano en el año 2000 se debieron a enfermedades no trasmisibles, siendo esta proporción semejante a la encontrada en zonas desarrolladas de Europa (86\%) y del Pacífico Occidental (75\%). Ese mismo año se produjeron solo en América Latina 119000 defunciones vinculadas con estilos de vida sedentarios. (CDC, 1996; Peña et al. 2000).

En México, la Encuesta Nacional de Saludy Nutrición(ENSANUT, 2006) reporta una prevalencia de sobrepeso en hombres y mujeres de 20 años y mas es de 42.5 y $37.4 \%$ mientras que la obesidad es de 34.5 y $24.2 \%$ respectivamente. En el mismo estudio la obesidad abdominal, definida como una cintura por arriba de los $102 \mathrm{~cm}$ para los hombres y mayor de $88 \mathrm{~cm}$ para las mujeres de acuerdo con el National Colesterol Education Papel (NCEP), muestra prevalencias de $24.1 \%$ en hombres y $61.9 \%$ en mujeres. Los resultados de la Encuesta Nacional de Salud de España 2006 revelan que aun que el 68,8\% de la población afirma que su estado de salud es bueno o muy bueno, y el 52,7\% de la población adulta presenta sobrepeso u obesidad. El papel del sedentarismo como factor de riesgo cardiovascular independiente ha sido muy estudiado en las últimas cuatro décadas (Kannel et al. 1979; Wingard et al. 1982; 


\begin{tabular}{|l|c|c|c|c|c|c|c|c|}
\hline \multicolumn{7}{|c|}{ Tabla 1. Características generales de los sujetos de estudio } \\
\hline Características generales & $\begin{array}{c}\text { Hombres } \\
\mathbf{( n = 3 2 )} \\
\text { Media }\end{array}$ & $\begin{array}{c}\text { UAS } \\
\text { México } \\
\text { Desviación } \\
\text { estándar }\end{array}$ & $\begin{array}{c}\text { Hombres } \\
\text { (n=11) } \\
\text { Media }\end{array}$ & $\begin{array}{c}\text { UHU } \\
\text { España } \\
\text { Desviación } \\
\text { estándar }\end{array}$ & $\begin{array}{c}\text { Mujere } \\
\mathbf{s} \text { (n=4) } \\
\text { Media }\end{array}$ & $\begin{array}{c}\text { UAS } \\
\text { México } \\
\text { Desviación } \\
\text { estándar }\end{array}$ & $\begin{array}{c}\text { Mujeres } \\
\text { (n=5) } \\
\text { Media }\end{array}$ & $\begin{array}{c}\text { UHU } \\
\text { España } \\
\text { Desviación } \\
\text { estándar }\end{array}$ \\
\hline Edad (años) & 42.9 & 7.4 & 43.2 & 9 & 42 & 7.34 & 29.8 & 5.2 \\
\hline Peso (kg) & 88.2 & 11.9 & 80.1 & 13.2 & 75 & 11 & 58.4 & 3.9 \\
\hline Estatura (cm) & 178.1 & 5 & 174.4 & 7.1 & 161.1 & .02 & 163.3 & 3.8 \\
\hline Circunferencia de Cintura (cm) & 98.4 & 3.9 & 92.3 & 10.6 & 89.1 & 11.7 & 72.6 & 4.5 \\
\hline IMC (kg/m2) & 29.78 & 3.93 & 26.2 & 3.9 & 28.85 & 5.21 & 22.1 & 1.3 \\
\hline
\end{tabular}

Paffenbarger et al. 1993; Rennie, et al. 2003). Los resultados de estos trabajos muestran un descenso de laprevalencia de enfermedades crónicas no trasmisibles como diabetes, obesidad, enfermedad cardiovascular, osteoporosis, síndrome metabólico e incluso algunas neoplasias, en aquellos sujetos físicamente activos. En México según el Programa Nacional de Cultura Física y Deporte 2001-2006, los hábitos de los mexicanos para realizar actividades físicas o deportivas se reduce a menos de una hora un día a la semana y menos del 7\% de la población mayor de 15 años realiza alguna actividad física o deporte que sea significativa para conservar su salud, mientras que en España el 59,6\% de la población adulta realiza ejercicio físico en su tiempo libre (un $63,1 \%$ de los hombres y el 56,3\% de las mujeres de 16 y más años). En los hombres, este hábito es más frecuente entre los 16 y 24 años y en las mujeres, entre los 45 y 64 años. (Encuesta Nacional de Salud de España 2006).

Los resultados anteriores son de encuestas poblacionales a nivel nacional de México y España discriminando poblaciones solo por el géneroy geográfia sin especificar características étnicas, socioeconómicas o grupos etéreos específicos por lo anterior, el presente estudio tiene como objetivo conocer prevalencia del nivel de actividad física, estado nutricio y obesidad abdominal en profesores universitarios de educación física de la Universidad Autónoma de Sinaloa (UAS) México y la Universidad de Huelva (UHU) siendo estos una población con un nivel deinstrucción elevado que junto con otras áreas del conocimiento forman recurso humano en el campo de la actividad física la cual a su vez coadyuva en la prevención y tratamiento de enfermedades crónico no trasmisibles.

\section{Material y métodos}

\subsection{Sujetos}

Como fase previa, para poder llevar a cabo el presente estudio fue necesario solicitar la autorización de los directivos de las instituciones educativas explicando de manera verbal y por escrito los propósitos y beneficios de la realización del presente estudio, así como el impacto del mismo. Los sujetos participantes fueron seleccionados a partir de una invitación personal autorizando participar voluntariamente. Se midió a 36 profesores de la Escuela Superior de Educación Física de la Universidad Autónoma de Sinaloa (se excluyeron tres sujetos una por embarazo y dos por no acceder a participar en el estudio) con una edad promedio de $42.8 \pm 7.3$ años, 32 de género masculino y 4 de género femenino, de Igual forma participaron 16 profesores del Departamento de Expresión Corporal y sus Didácticas de la Facultad de Educación de la Universidad de Huelva, con una edad promedio de 39.1 \pm 9.8 años de edad, 11 hombres y 4 mujeres (se excluyo una por embarazo).

\subsection{Instrumentos y procedimientos}

A cada sujeto se le tomo, las medidas antropométricas, de peso y talla determinándose mediante una bascula digital con capacidad de 140 Kg y una precisión de 100 g y estadiómetro marca SECA, se uso cinta métrica metálica inextensible tipo Lufking de 2 metros de largo para la obtención de la circunferenciaumbilical. Para estimar el nivel de actividad física se usó el formato corto, versión en español, del cuestionario internacional de actividad física IPAQ(Craig, et al. 2003). Para el análisis de los datos se utilizó el programa SPSS Versión 13.0 calculando el índice de masa corporal IMC $=\left(\mathrm{kg} / \mathrm{m}^{2}\right)$, clasificándose por los criterios de la (OMS) con valores $<18.5$ el peso bajo, $>18.5 \mathrm{y}<$ a 24.9 peso normal, $>25$ y $<29.9$ sobrepeso y $>30$ obesidad. La circunferencia de cintura (cm) bajo los criterios de la NCEP como riesgo para desarrollar complicaciones metabólicas relacionadas con la obesidad se clasificaron los valores $>102 \mathrm{~cm}$ en hombres y $>88 \mathrm{~cm}$ en mujeres como riesgo sustancialmente aumentado, los valores e» $94 \mathrm{y}<101.9$ en hombres $\mathrm{y}$ e» 80 y $<88$ como riesgo aumentado y los valores $<94$ en hombres $y$ $<80$ en mujeres se clasificaron como riesgo bajo. Mediante el cuestionario IPAQ se clasifico esta variable en tres niveles, alta, moderada y baja, en función del valor de los MET's. Con los resultados obtenidos se elaboro una base de datos en el programa SPSS Versión 13.0 para su procesamiento estadístico.

\section{Resultados}

Los resultados que se presentan a continuación son de los 52 profesores evaluados siendo 36 de la Universidad Autónoma de Sinaloa (32 hombres y 4 mujeres) con una edad promedio de $42.8 \pm 7$.3 años y 16 de la Universidad de Huelva ( 11 hombres y 4 mujeres) con una edad promedio de edad promedio de $39.06 \pm 10.1$ años, en la tabla 1 se pueden observar las características generales de los profesores. donde en los de género masculino de las dos universidades estudiadas se resalta que en promedio los profesores de la UAS y UHU presentan índices de masa corporal que se clasifican como sobrepeso, y en el caso de la circunferencia de cintura los profesores de la UAS presentan en promedio una clasificación de riego sustancialmente aumentado mientras que los de la UHU riesgo aumentado. Se observa también en la tabla 1 las características generales de las profesoras de ambas universidades, donde solo presentan promedio de rangos óptimos en estado nutricioy obesidad abdominal (normal y bajo respectivamente) en las profesoras de la UHU por lo contrario rangos de riesgo (sobrepeso y sustancialmente aumentado) en estado nutricio y obesidad abdominal en las profesoras de la UAS.

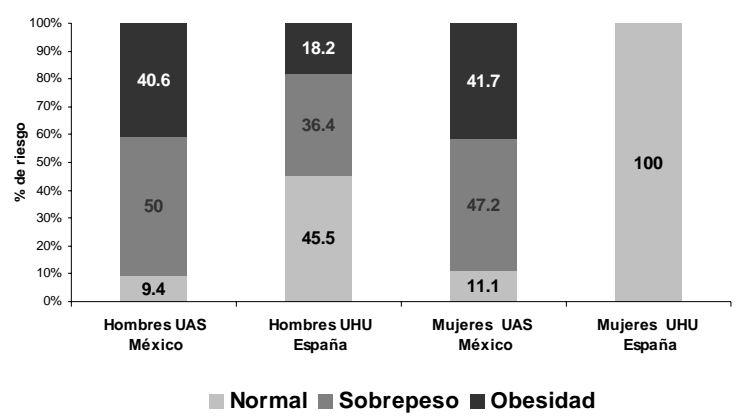

Figura 1. Comparativo de distribución porcentual del estado nutricio de la población por género 


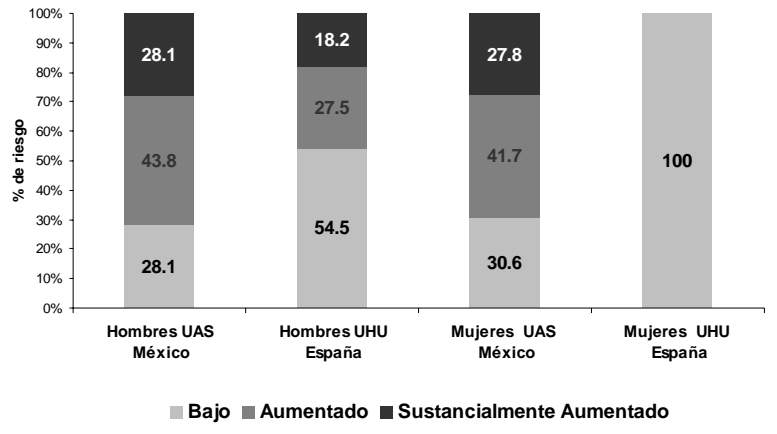

Figura 2. Comparativo de distribución porcentual de la obesidad abdominal de la población por género

En la figura 1 se muestra la distribución porcentual del IMC clasificado por la OMS del total de profesores por género. En ninguno de los sujetos estudiados se encontró estado nutricio bajo el cual es un indicador de desnutrición, de lo contrario el sobrepeso y obesidad se observo en mayor medida los hombres y mujeres de la UAS con $90.6 \%$ y 88.9\% respectivamente, cabe resaltar que las mujeres de la UHU solo se presenta un estado nutricio normal.

En la figura2se observa la distribución porcentual dela circunferencia decintura (obesidadabdominal), clasificada según suriesgopara desarrollar complicaciones metabólicas relacionadas a la obesidad en el total de profesores por género, presentándose en mayor proporción riesgos sustancialmente aumentados en los hombres y mujeres de la UAS (28.1\% y 27.8\%), mas de la mitad de los profesores de género masculino de la UHU presentaron riesgo bajo mientras que en el total de las mujeres se encontró con riesgo bajo.

La figura 3 describe la distribución porcentual del nivel de actividad física de los profesores por género, de acuerdo a los criterios del IPAQ, en la se observan patrones porcentuales similares al combinar el nivel de actividad física alta y moderada, fluctuando de rangos entre el $68.8 \%$ y $80 \%$.

\section{Discusión}

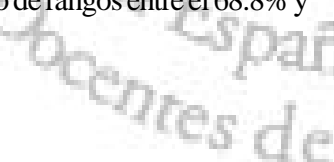

La primera estrategia para contener el problema de la obesidad es diagnosticar el problema identificando en al menos algunos de los factores modificables y asociados con esta. De acuerdo con lo resultados obtenidos podemos decir que los profesores de la UAS se observan grandes problemas en relación a la presencia de sobrepeso y obesidad presentándose en estos en 90.6 \% de sus casos, siendo esta considerablemente más alta que la reportada a la media nacional(69.3\%) en adultos mexicanos según los reportes de la más reciente encuesta nacional de salud realizada en México (ENSANUT 2006). Y aunque en los profesores de la UHU, la problemática de sobrepeso y obesidad es menor (54.6\%) de igual forma rebasa el porcentaje promedio de la población adulta española (52,7\%) según datos de la Encuesta Nacional de Salud de España 2006 (utilizándose la misma metodología para ambas encuestas).

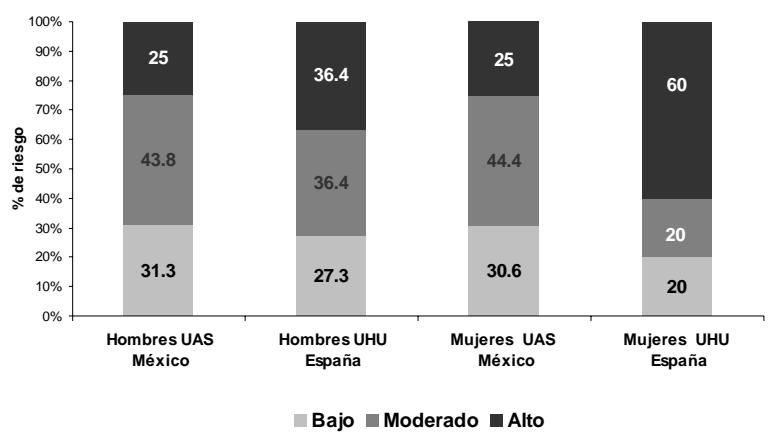

Figura 3. Comparativo de distribución porcentual del nivel de actividad física de la población por género
La obesidad abdominal, en los profesores de la UHU se observó con mas baja presencia respecto a los de la UAS, resultando el género masculino con el mismo patrón epidemiológico de obesidad, siendo este mayor (28.1\%) a la media nacional (24.1\%) de los hombres mexicanos, de lo contrario las mujeres (27.8\%) presentaron prevalencias mas bajas a las reportadas en adultas mexicanas $61.9 \%$.

Respecto al nivel de actividad física se puede observar gran similitud entre los profesores de ambas universidades reportando $75.1 \%$ de combinación de nivel de actividad física alta y moderada en los profesores de la UHU y $69.4 \%$ por parte de los profesores de la UAS, estableciéndose que la población estudiada poca presencia de sedentarismo, aunque el nivel de actividad física de los sujetos evaluados contrasta con los resultados encontrados en relación a los altos porcentajes de sobrepeso y obesidad.

Una investigación utilizando el cuestionario IPAQ como herramienta de medición en México reportan menor nivel de actividad física como el que nos muestra la ENSANUT 2006 realizado en adolescentes de 10 a 19 años de edad, el $35.2 \%$ resulto con un nivel de actividad física alta, el $24.4 \%$ moderada, el y $40.4 \%$ con un nivel de actividad física baja. Los niveles de actividad física de los sujetos estudiados contrastan con los resultados encontrados en estudios poblacionales utilizando la misma metodología del cuestionario IPAQ, llevado acabo en ciudades latinoamericanas por ejemplo resultados de Gómez et al. (2005) muestran que en adultos de Bogota, Colombia se presento una prevalecía del nivel de actividad física alta y moderada de $63.6 \%$, menor a la reportada en esta investigación de igual manera, Matsudo et al. (2000) refieren en adultos de Sao Pablo, Brasil, una prevalencia de nivel de actividad física alta y moderada de $46.5 \%$.

Otro estudio poblacional en adultos mexicanos de prevalencia de actividad física pero utilizando otra metodología clasificándola a la actividad física de acuerdo con su intensidad, frecuencia y duración concluyeron que la prevalencia de actividad física recomendada es baja y la de inactividad física, alta. (Acosta-Cázarez et al. 2006). Un estudio realizado en la región de Murcia en adultos españoles refiere que solo el $17.8 \%$ realiza actividad física deportiva intensa e» 2 horas a la semana. (Martínez-Ros et al. 2003).

Una investigación realizada en sujetos similares (Profesores del Instituto de Enseñanza Media de la Universidad de Salta, Argentina), reportan un marcado sedentarismo aunque no se uso la misma metodología (Cruceiro et. al. 2007). Otra investigación realizada en profesores universitarios de la Universidad Federal de Santa Catarina Brasil donde semiden factores y barreras haciala actividad física, concluyó que el nivel de actividad física de estos fue reducido e irregular y la barrera principal hacia la actividad física fue la falta de tiempo (De Oliveira Martins, 2000).

El tipo de investigación transversal se puede identificar como limitación en el estudio, ya que no permite establecer relaciones de causalidad, al no medir variables asociadas a la obesidad y el nivel de actividad física, aun que en el caso de el estado nutricio y obesidad abdominal se realizaron usando la misma metodología facilitando así criterios comparativos, los resultados de este estudio pueden servir de argumento para analizar otras poblaciones de semejantes características con la finalidad de desarrollar una descripción adecuada del estado nivel de actividad física, estado nutricio y obesidad abdominal de profesores delárea de educación física.

\section{Conclusiones}

En los resultados se observa que los docentes presentan prevalencias de sobrepeso y obesidad muy altas, inclusive mas altas que la media nacional de su país contrastando con el nivel de actividad física que reportan. Tomando en cuenta los factores relacionados a enfermedades crónico no trasmisibles es de esperar que los profesionales de la salud (dada su formación académica y como modelo social) presenten menores factores de riesgo a al sobrepeso y obesidad. En estos sujetos es urgente aplicar medidas que conduzcan a la prevención y tratamiento de la obesidad. 


\section{Agradecimientos}

A la asociación Universitaria Iberoamericana de Posgrado (AUIP) a través de su programa de movilidad académica entre instituciones asociadas a laAUIP, así como a la Dirección de Intercambioy Vinculación Académica de la UniversidadAutónoma de Sinaloa por el financiamiento de la presente investigación, y a la valiosa participación de los profesores de Educación Física de la Escuela Superior de Educación Física de la Universidad Autónoma de Sinaloa y el Departamento de Expresión Musical, Plástica, Corporal y sus Didácticas dela Facultad deEducación de la Universidad de Huelva que accedieron voluntariamente a ser parte de esta investigación.

\section{Referencias bibliográficas}

Acosta-Cázares, B, Aranda-Álvarez, J, Reyes-Morales, H (2006) ENCOPREVENIMSS 2004 Patrones de actividad física de la mujer y del hombre. Revista Médica del Instituto Mexicano del Seguro Social, 44 (11): 79-86.

CENTER FOR DISEASE CONTROL AND PREVENTION (1996) Neighborhood Safety and the Prevalence of Physical InactivitySelected Status. Journal of the American Medical Association, 281, 15.

COMISIÓN NACIONAL DEL DEPORTE (2001) Programa Nacional de Cultura Física y Deporte 2001-2006, México. (En línea). http://www.conade.gob.mx/paginas_07/doc_basicos.asp (consulta: 10 de agosto 2007).

Craig, CL, Marshall, AL., Sjostrom, M, Bauman, AE, Booth, ML, Ainsworth, BE (2003) International physical activity questionnaire: and Exercise, 35: 1381-95.

De Oliveira Martins, M (2000) Estudo dos Fatores Determinantes da Prática deAtividades Físicas de Professores Universitários. Revista Brasileira de Cineantropometria \& Desempenho Humano, 2 (1): 112. Valdiviezo, MS, Soruco A(2007) Estilos de vida de profesores del Instituto de Enseñanza Media de la Universidad Nacional de Salta. República Argentina. Revista de Salud Pública y Nutrición, 8:1. de actividad física global en la población adulta deBogotá(Colombia). Prevalencia y factores asociados. Gaceta Sanitaria. 19:3.

Kannell, WB, Sorlie, P(1979) Some health benefits of physical activity. The Framingham Study. Archives of Internal Medicine, 139: 857 861.

Lang, T, Ducimetiere, P(1995) Premature Cardiovascular Mortality in France: Divergent Evolution between Social Categories from 1970 to 1990. International Journal of Epidemiology, 24: 331-339. 12-country reliability and validity. Medicine and Science in Sports

Couceiro, M, Passamai, M, Contreras, N, Villagrán, E, Zimmer, M,

Gómez, L, Duperly, J, Lucumí, D, Gámez, R, Venegas, A(2005) Nivel

Lynch, JW, Kaplan, FA, Cohen, RD, Tuomilehto, J, Salonen, JT(1996) Do cardiovascular risk factors explain the relation between socioeconomic status, risk of all cause mortality, cardiovascular mortality and acute myocardial infarction. American Journal of Epidemiology, 144: 934-942.

Martínez-Ros, M.T, Tormo, M.J, Pérez-Flores, D, Navarro, C (2003) Actividad física deportiva en una muestra representativa de la población de la Región de Murcia. Gaceta Sanitaria, 17:1.

Matsudo, SM, Matsudo, V,Araújo, T, Andrade, D, Andrade, E, Oliveira, L (2000) Nível de atividade física da população do Estado de São Paulo: análise de acordo com o gênero, idade, nível socioeconômico, distribuição geográfica e de conhecimento. Revista Brasileira do. Ciências e Movimiento, 10:41-50.

MINISTERIO DE SANIDAD Y CONSUMO (2007) Encuesta Nacional de Salud de España 2006. (En línea). http://www.msc.es/ estadEstudios/estadisticas/encuestaNacional/encuestaNac2006/ EstilosVidaPorcentaje.pdf (consulta: 10 de enero 2008).

ORGANIZACIÓN MUNDIAL DE LA SALUD (1997). Obesity: Prevening and Managing the Global Epidemic of obesity. Report of the WHO consulation of obesity. Geneve june 1997. (En línea). http: / / w w w. who.int/bookorders/anglais / detart1.jsp?sesslan=1\&codlan=1\&codcol=10\&codcch=894\# (consulta: 6 de junio 2007).

ORGANIZACIÓN PANAMERICANA DE LA SALUD (2003) Consulta Regional OPS sobre Alimentación Saludabley Actividad Física en las Américas. (En línea). http://www.dpaslac.org/uploads/ 1154103060.pdf (consulta: 6 de junio 2007).

Paffenbarger, R, Hyde, R, Wing, A, Lee, I, Jung, D, Kampter, J (1993) The association of changes in physical activity level and other lifestyle characteristics with mortality among men. New England Journal of Medicine, 328:538-545.

Peña, M, Bacallao, J(2001) «La obesidad y sus tendencias en la región». Revista Panamericana Salud Publica, 10 (2): 75.

Rennie, KL, McCarthy, N, Yazdgerdi, S, Marmot, M, Brunner, E (2003) Association of the metabolic syndrome with both vigorous and moderate physical activity. International Journal of Epidemiology, 32: 600-606.

Rivera-Dommarco, J, Cuevas, L, Shamah-Levy, T, Villalpando, S, Avila, M, Jiménez, A (2006) Encuesta Nacional de Salud y Nutrición 2006. (Estado Nutricio) (En línea). http://www.insp.mx/ensanut/ ensanut2006.pdf (consulta: 22 julio 2007).

Shamah-Levy, T, Morales, C, Rivera-Dommarco, J, Hernández, B., Gómez, LM, Mauricio, ER, Mendoza, A, Villalpando, S (2006) Encuesta Nacional de Salud y Nutrición 2006. (Actividad física en adolescentes) (En línea). http://www.insp.mx/ensanut/ ensanut2006.pdf (consulta: 16 agosto 2007).

Wingard, DL (1982) The sex differential in mortality rates: demographic and behavioral factors. American Journal of Epidemiology, 115: 205-216.
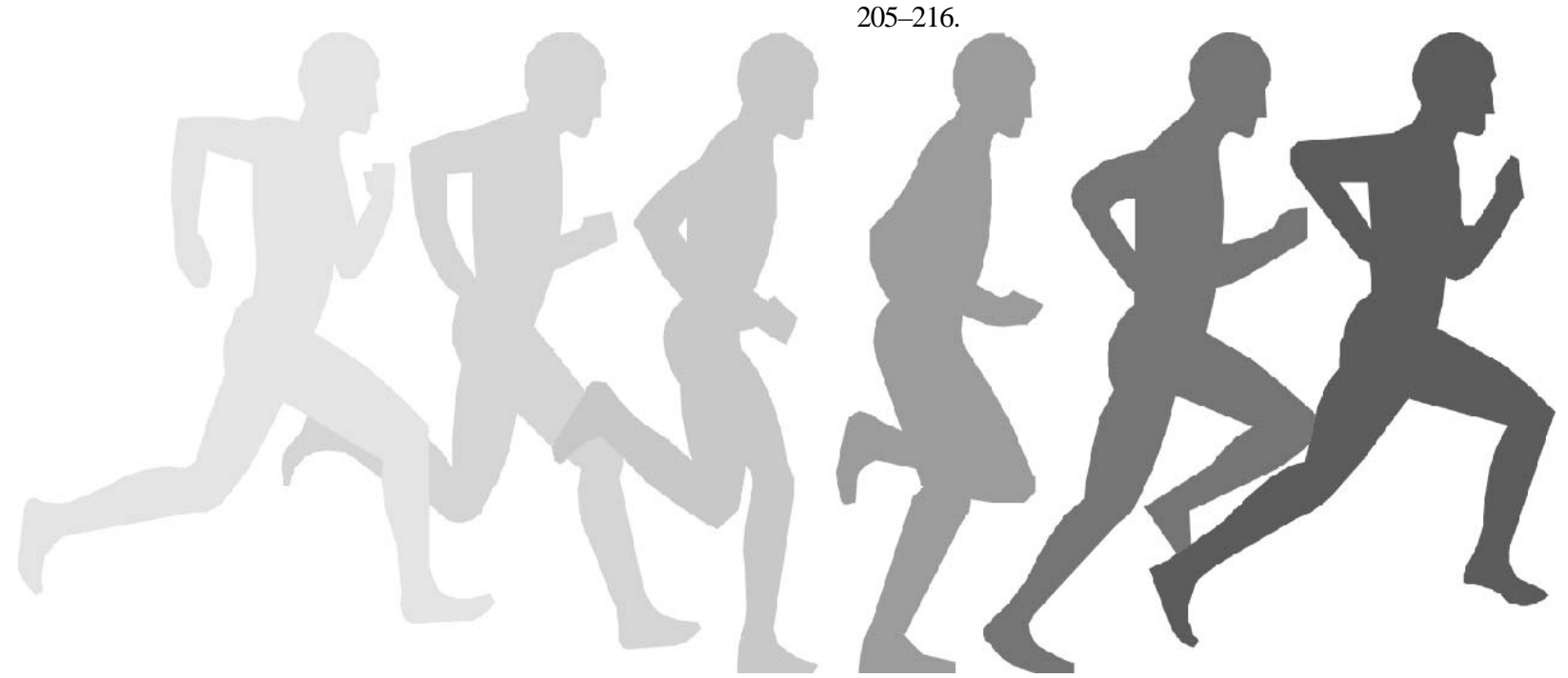